\title{
The Quest to Make a Ferromagnet with Cold Atoms
}

\section{Experimentalists working with cold atoms are closing in on the famous Stoner model of ferromagnetism.}

\section{by Lindsay LeBlanc*}

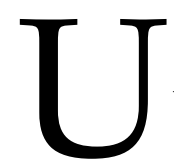
ltracold gases of fermionic atoms (Fermi gases) offer vast opportunities to explore many-body behavior. With modern experimental tools, researchers can make the interaction between the atoms attractive or repulsive, and they can tune the interaction strength over several orders of magnitude. A long-time goal has been to use this tunability to drive the atoms into a ferromagnetic state, in which repulsive interactions cause the atoms to point their magnetic moments in the same direction. The realization of a cold-atom ferromagnet would provide a unique chance to examine the microscopic mechanisms behind magnetism, and to benchmark theoretical models. However, the atoms in these experiments tend to rapidly combine into weakly bound molecules, which

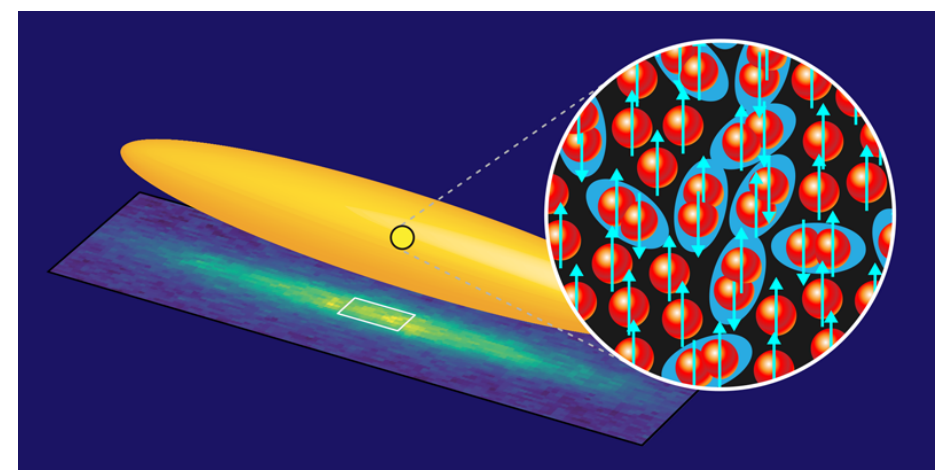

Figure 1: Experimentalists have long been trying to observe Stoner ferromagnetism in ultracold gases of fermionic atoms. Using a novel experimental approach, Zaccanti and collaborators observed signatures of ferromagnetism in a cold gas of lithium atoms (yellow). But this state came with a twist, as the magnetically coupled atoms were found to co-exist with molecules in a state that resembles a micro-emulsion (inset). (APS/Alan Stonebraker)

*Department of Physics, University of Alberta, Edmonton, AB, Canada obscures the magnetic behavior. Matteo Zaccanti of the European Laboratory for Non-Linear Spectroscopy and the University of Florence, Italy, and colleagues have now developed a new technique that discriminates the atomic signal from that of the molecules, allowing them to observe the emergence of magnetic interactions between the atoms that are indicative of a ferromagnetic phase [1].

Much remains to be understood about magnets at a microscopic level, such as what causes the long-range ordering of magnetic moments. Ultracold Fermi gases, in which twolevel atoms mimic spin-1/2 electrons in a magnetic solid, allow real-life simulations that address this question and others. Experimentalists set up a certain interaction between the atoms and then monitor the gas as the atoms evolve into an ordered phase. A key advantage of such quantum "simulators" is that they avoid the computational difficulties associated with theoretical many-body models, particularly with fermionic particles like electrons [2].

One magnetism model that has seemed particularly well suited for simulation with atoms is Edmund Stoner's 1933 model of an itinerant ferromagnet [3], which applies to a gas of free electrons with repulsive interactions between them. When the interaction strength reaches a threshold value, the randomly oriented electron spins align themselves; that is, they transition into a ferromagnetic state. Since identical electrons-obeying Pauli's exclusion principle-can't occupy the same quantum state, electrons with aligned spins have to spread out over a broader range of energy levels. Although this increases their kinetic energy, it eliminates the interaction energy, which, for large enough interaction strength, overbalances the kinetic energy cost. Stoner's mean-field treatment of this situation showed that the ferromagnetic instability occurs when $k_{F} a=\pi / 2$, where $a$ is a measure of the interaction strength, and $k_{F}$, the Fermi wave vector, is a measure of the particle density.

A 2005 proposal for simulating Stoner ferromagnetism with ultracold fermionic atoms triggered a series of experiments [4]. The idea was to tune the interaction strength $a$ between the atoms using a Feshbach resonance, where an external magnetic field creates a resonance between free atoms and bound states. In these early experiments, researchers observed a suppression of atomic collisions with 
an increase in repulsive interaction strength [5]. This reduction in correlations, or anticorrelations, was interpreted as the onset of Stoner-like ferromagnetism, since electrons with the same spin orientation are forbidden to interact because of Pauli's principle. However, researchers soon realized that these observations could also be explained by the formation of paired states of atoms [6]. Subsequent experiments were careful to account for the contamination from paired atoms. These experiments either isolated the atomic signal, or they started with a population of atoms with the same spin orientation and then probed the stability of this "artificial" ferromagnetism over time (see, for example, Refs. [7-9]). Such measurements inferred some form of ferromagnetism from signatures of anticorrelations between individual atoms, but the tendency of the atoms to form molecules continued to be a persistent complication.

In their paper, Zaccanti and colleagues took a new approach to observing ferromagnetism in cold fermionic atoms. As in the previous experiments, they created a Feshbach resonance in order to subject lithium atoms in a gas to repulsive interactions, and they performed radio frequency spectroscopy on the gas [10]. The resulting spectrum has two distinct peaks, one from the molecular states, and one from the repulsively interacting atoms; the shape and position of this second peak carry information about the atomic correlations. Such spectra have been measured before, but the team incorporated their spectral measurement into a "pump-probe" experiment. Specifically, they "pump" the gas by initiating repulsive interactions among the atoms, and then, after a time delay, they "probe" the gas by measuring the radio frequency spectrum. This approach allows the researchers to track the time evolution of the atomic peak and the buildup of anticorrelations over short timescales.

Thanks to the time resolution of their approach, the authors found that anticorrelations among the atoms indicative of magnetism do indeed emerge spontaneously above a threshold interaction strength. Moreover, these anticorrelations grow about twice as fast as the molecules form, which made the measurement of the atomic signal possible. In addition, anticorrelations among the individual repulsive fermions were seen to persist on a timescale that was long compared to when the anticorrelations set in. Further, the researchers found that the molecular pairs and the atoms in the quantum gas were segregated in spatially distinct regions, forming a glassy state resembling a microemulsion of pairs dispersed in the sea of single atoms (Fig. 1).

In many ways, Zaccanti and colleagues have come closer than ever before to seeing ferromagnetism as described by the Stoner model: they see the real-time onset of magnetic anticorrelations as the atomic spins align. But the mixture of phases they achieve is far from the simple ferromagnetic phase Stoner predicted. The team's findings are a reminder that, for all of the celebrated precision and versatility of ultracold atomic systems, we must be careful and cognizant of their limitations. At the outset, the problem of itinerant ferromagnetism seemed like a good match to ultracold fermions. As we have since learned, the simple analogy to Stoner ferromagnetism just isn't available because these repulsive Fermi gases will always be infiltrated by molecules. However, as experiments like this one show, persistence and ingenuity pay off: new kinds of many-body correlations such as quantum emulsions could be lurking in this system, interesting in and of themselves. Also, with new techniques come fresh possibilities for studying the complex and hardto-predict behavior that have made many-body systems so fascinating to study.

This research is published in Physical Review Letters.

\section{REFERENCES}

[1] A. Amico et al., "Time-resolved observation of competing attractive and repulsive short-range correlations in strongly interacting Fermi gases," Phys. Rev. Lett. 121, 253602 (2018).

[2] I. Bloch, J. Dalibard, and S. Nascimbène, "Quantum simulations with ultracold quantum gases," Nat. Phys. 8, 267 (2012).

[3] E. C. Stoner, "LXXX. Atomic moments in ferromagnetic metals and alloys with non-ferromagnetic elements," Philos. Mag. 15, 1018 (1933).

[4] R. A. Duine and A. H. MacDonald, "Itinerant ferromagnetism in an ultracold atom Fermi gas," Phys. Rev. Lett. 95, 230403 (2005).

[5] G.-B. Jo, Y.-R. Lee, J.-H. Choi, C. A. Christensen, T. H. Kim, J. H. Thywissen, D. E. Pritchard, and W. Ketterle, "Itinerant ferromagnetism in a Fermi gas of ultracold sstoms," Science 325, 1521 (2009).

[6] S. Pilati, G. Bertaina, S. Giorgini, and M. Troyer, "Itinerant ferromagnetism of a repulsive atomic Fermi gas: A quantum Monte Carlo study," Phys. Rev. Lett. 105, 030405 (2010).

[7] C. Kohstall, M. Zaccanti, M. Jag, A. Trenkwalder, P. Massignan, G. M. Bruun, F. Schreck, and R. Grimm, "Metastability and coherence of repulsive polarons in a strongly interacting Fermi mixture," Nature 485, 615 (2012).

[8] A. B. Bardon et al., "Transverse demagnetization dynamics of a unitary Fermi gas," Science 344, 722 (2014).

[9] G. Valtolina, F. Scazza, A. Amico, A. Burchianti, A. Recati, T. Enss, M. Inguscio, M. Zaccanti, and G. Roati, "Exploring the ferromagnetic behaviour of a repulsive Fermi gas through spin dynamics," Nat. Phys. 13, 704 (2017).

[10] F. Scazza, G. Valtolina, P. Massignan, A. Recati, A. Amico, A. Burchianti, C. Fort, M. Inguscio, M. Zaccanti, and G. Roati, "Repulsive Fermi polarons in a resonant mixture of ultracold ${ }^{6}$ Li atoms," Phys. Rev. Lett. 118, 083602 (2017).

10.1103/Physics.11.131 